\title{
Association Between Excessive Daytime Sleepiness and Functional Gastrointestinal Disorders: A Population-based Study in China
}

\author{
Sicheng Wu, Shuqi Chen, Yanfang Zhao, Xiuqiang Ma, Rui Wang, and Jia He* \\ Department of Health Statistics, Second Military Medical University, Shanghai, China
}

\begin{abstract}
Background/Aims
Several studies have demonstrated that sleep problems are associated with functional gastrointestinal disorders (FGIDs): irritable bowel syndrome (IBS), functional dyspepsia, etc, but the relationship between excessive daytime sleepiness (EDS) and FGIDs has not been systematically studied in the general population. This study aims to explore the relationship between EDS and specific types of FGIDs and the effect of the number of FGIDs on EDS.
\end{abstract}

\section{Methods}

A sample of 3600 individuals (aged 18-80 years) was selected from 5 regions in China using a randomized, stratified, multi-stage sampling method. EDS was measured by the Epworth Sleepiness Scale, while gastroesophageal reflux disease (GERD) and other FGIDs were assessed by Reflux Disease Questionnaire and the Rome II diagnostic criteria, respectively.

\section{Results}

The survey was completed by 2906 individuals (response rate: 80.72\%), and 644 individuals (22.16\%) had EDS. EDS was significantly associated with ulcer-like dyspepsia (OR, 2.50; 95\% Cl, 1.08-5.79), diarrhea-predominant IBS (OR, 2.00; 95\% Cl, 1.09-3.66), alternating IBS (OR, 2.32; 95\% Cl, 1.30-4.13), functional constipation (OR, 1.68; $95 \% \mathrm{Cl}, 1.20-2.35)$, and GERD (OR, 1.72; $95 \% \mathrm{Cl}$, 1.08-2.72). Risk of EDS increased along with the increasing numbers of FGIDs: with 1 FGID (OR, 1.72; 95\% Cl, 1.37-2.15); with 2 FGIDs (OR, 2.43; $95 \% \mathrm{Cl}, 1.63-3.62)$; and with 3 or more FGIDs (OR, 3.26; $95 \% \mathrm{Cl}, 1.37-7.78)$.

\section{Conclusions}

FGIDs, such as ulcer-like dyspepsia, diarrhea-predominant IBS, alternating IBS, functional constipation, and GERD, were significantly associated with EDS. Those who suffered from more kinds of FGIDs were more susceptible to EDS.

(J Neurogastroenterol Motil 2017;23:298-305)

\section{Key Words}

Epidemiology; Excessive somnolence disorders; Functional gastrointestinal disorders

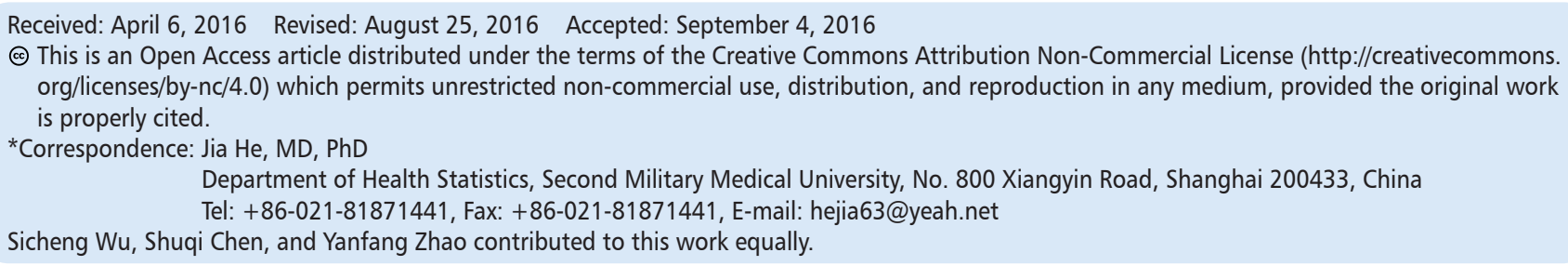




\section{Introduction}

Excessive daytime sleepiness (EDS) is a symptom of inability to stay awake and alert during major waking episodes of the day. Prevalence of EDS in the general population varies among different countries ranging from $2.5 \%$ to $22.2 \% .^{1-6}$ EDS can result in psychosocial distress, decreased work or school performance, impairment of health-related quality of life (HRQOL), and higher risk for accidents and occupational injuries. ${ }^{6-8}$ Besides common causes of EDS such as sleep deficiencies, drugs, depression and migraines, obesity, diabetes, and ulcers were also supposed to be significantly associated with EDS.,

It was reported that sleep problems, such as sleep impairment, sleep disorders, and sleep disruption were associated with functional gastrointestinal disorders (FGIDs), such as functional dyspepsia (FD) and irritable bowel syndrome (IBS). ${ }^{10-15}$ As a sleep related symptom, EDS was also found to be associated with FGIDs. In China, EDS was significantly more prevalent among participants with gastroesophageal reflux disease (GERD) than those without. ${ }^{16}$ Presence of FD and IBS symptoms in addition to GERD symptoms exhibited a strong relationship with daytime sleepiness in Japanese. ${ }^{17}$ In Israel, IBS patients tended to suffer from daytime sleepiness measured by the Epworth Sleepiness Scale (ESS). ${ }^{18}$

EDS is a common sleep-related symptom in China, it is more prevalent in the general Chinese population (22.2\%) than in general American (8.7\%) and Japanese populations (2.5\%). ${ }^{4-6}$ It is of interest whether EDS was associated with FGIDs. However, the relationship between EDS and FGIDs in the general population has not been systematically studied, especially in China.

In this study, we aim to (1) evaluate the association between EDS and specific types of FGIDs considering a variety of factors, and (2) assess the effect of the number of FGIDs on EDS using extracted data from the Systematic Investigation of Gastrointestinal Diseases in China (SILC) data pool.

\section{Materials and Methods}

\section{Study Design and Sample}

As part of the SILC study, detailed design and methodology of which were described elsewhere. ${ }^{19}$ In the SILC study, a total of 18000 adults aged from 18-80 were selected from Shanghai, Beijing, Xi'an, Wuhan, and Guangzhou (the 5 cities are located in Eastern, Northern, Western, Middle, and Southern China respec- tively) using a randomized, stratified, and multi-stage sampling methodology. Firstly, we treated the urban and rural population as 2 separate strata. Then for the urban stratum, a 3-stage sampling was performed in the sequence of districts, blocks, and residential, for the rural stratum, the 3-stage sampling was performed in the sequence of counties, townships, and villages. All residents of the selected residential areas or villages were stratified according to their age and sex, individuals were randomly selected from these strata in proportion to the overall age and sex distribution of the population in that region, using data from recent government population surveys. Residents who were not in the 18-80 age group, or suffering from psychiatric illnesses were excluded from the study. The fieldwork period was from April 2007 to January 2008. Questionnaires were self-administered, with trained and supervised facilitators available to explain any questions that respondents found unclear. ${ }^{19}$ The study was approved by the Second Military Medical University Ethics Committee. All respondents signed a written informed consent before participation.

\section{Measurements}

A general information questionnaire was used to collect information including gender, age, marital status, education level, family income, occupation, lifestyle habits (including smoking status, alcohol consumption, and frequency of recreational exercise), and family history of gastrointestinal diseases, whether they had been diagnosed by physicians with the following chronic diseases: liver disease, hypertension, myocardial infarction, angina pectoris, cerebrovascular disease, chronic bronchitis, asthma, renal disease, osteoarthritis, rheumarthritis, and diabetes. ${ }^{19}$ Body mass index (BMI) was calculated using height and weight measured by trained investigators, then the BMI was divided into 4 groups according to the Chinese BMI categories. ${ }^{20}$ The Chinese version of ESS, modified Chinese version of the Rome II questionnaire (gastroduodenal, bowel, and biliary items only) and the Chinese version of Reflux Disease Questionnaire (RDQ) were applied to measure EDS, FGIDs, and GERD. ${ }^{6,16,21-23}$ The ESS is an 8-item, self-administered questionnaire covering eight situations commonly encountered in daily life. Each participant was instructed to answer how likely it is that he/she would fall asleep in different situations by giving a score ranging from 0 to 3. Thus, the total score of the ESS ranges from 0 to $24{ }^{6}$ Higher ESS scores mean that the subject is sleepier during daytime and ESS score greater than 10 indicates significant sleepiness. ${ }^{24}$ We defined subjective EDS as the ESS score greater than $10 .^{21}$ Response rate, reliability and validity of the Mandarin version of ESS have been validated before. ${ }^{6}$ Explanation of Chinese version of 
the Rome II questionnaire and RDQ has been reported in previous studies. $^{16,25}$

All participants completed Chinese versions of the RDQ and the modified Rome II questionnaire; a random sub-sample of the total sample (20\% in each region, total 3600 participants) were also invited to fill in the 36-item Short Form Health Survey and Epworth Sleepiness Scale (ESS). ${ }^{19}$

\section{Data Collection}

In our study, records of the $20 \%$ sub-samples were extracted for analysis purposes from the SILC study data pool.

\section{Statistical Methods}

SAS 9.4 software (SAS Institute, Cary, NC, USA) was used for the data analyses. Median (Q1, Q3) were applied to describe asymmetrically distributed data, such as the ESS score. Odds ratio (OR) and $95 \%$ confidence interval $(95 \% \mathrm{CI})$ were calculated by multivariate logistic regression. All hypothesis tests used two-sided tests, and $P$-values of less than 0.05 were considered statistically significant.

\section{Results}

\section{Demographic Characteristics of Excessive Daytime Sleepiness}

Among the sub-sample of 3600 participants, a total of 2906 individuals completed the survey (response rate: $80.72 \%$ ), and 644 individuals $(22.16 \%$ ) had EDS (defined as ESS > 10). Demographic characteristics of the individuals with EDS, and their association with EDS are shown in Table 1. EDS was significantly more common in the overweight group (defined as BMI in the range of $\left.24-27.9 \mathrm{~kg} / \mathrm{m}^{2}\right)(\mathrm{OR}, 1.27 ; 95 \% \mathrm{CI}, 1.02-1.57)$ and in the obesity group (defined as BMI great than $\left.28.0 \mathrm{~kg} / \mathrm{m}^{2}\right)(\mathrm{OR}, 1.68$; 95\% CI, 1.20-2.35), compared with those with BMI in the normal range of $18.5-23.9 \mathrm{~kg} / \mathrm{m}^{2}$. EDS was significantly more common in those with education level of primary school or lower (OR, 1.68; $95 \% \mathrm{CI}, 1.27-2.24)$ and in those with education level of university or higher (OR, 1.68; 95\% CI, 1.29-2.17), compared with those with education level of secondary/high school. We did not observe significant association between EDS and region, gender, age,

Table 1. Distribution of Demographic Characteristics According to Excessive Daytime Sleepiness in the Chinese population

\begin{tabular}{|c|c|c|c|}
\hline \multirow{3}{*}{ Characteristics } & \multirow{3}{*}{ Subjects (n [\%]) } & \multicolumn{2}{|c|}{ Subjects with EDS (ESS > 10) } \\
\hline & & \multirow{2}{*}{$\mathrm{n}(\%)$} & Multivariate analysis \\
\hline & & & OR $(95 \% \mathrm{CI})$ \\
\hline \multicolumn{4}{|l|}{ Gender } \\
\hline Female & $1503(51.72)$ & $336(22.36)$ & 1.00 \\
\hline Male & $1403(48.28)$ & $308(21.95)$ & $0.89(0.69-1.15)$ \\
\hline \multicolumn{4}{|l|}{ Region } \\
\hline Urban & $1544(53.13)$ & $339(21.96)$ & 1.00 \\
\hline Rural & $1362(46.87)$ & $305(22.39)$ & $1.05(0.86-1.28)$ \\
\hline \multicolumn{4}{|l|}{ Age (yr) } \\
\hline $18-29$ & $717(24.67)$ & $161(22.45)$ & $1.23(0.89-1.71)$ \\
\hline $30-39$ & $695(23.92)$ & $153(22.01)$ & 1.00 \\
\hline $40-49$ & $688(23.68)$ & $148(21.51)$ & $0.98(0.75-1.28)$ \\
\hline $50-59$ & $424(14.59)$ & $83(19.58)$ & $0.77(0.56-1.06)$ \\
\hline $60-80$ & $382(13.15)$ & $99(25.92)$ & $0.81(0.56-1.18)$ \\
\hline \multicolumn{4}{|l|}{$\operatorname{BMI}\left(\mathrm{kg} / \mathrm{m}^{2}\right)^{\mathrm{a}}$} \\
\hline$<18.5$ & $243(8.37)$ & $56(23.05)$ & $1.11(0.79-1.56)$ \\
\hline $18.5-23.9$ & $1714(59.06)$ & $348(20.30)$ & 1.00 \\
\hline $24.0-27.9$ & $732(25.22)$ & $175(23.91)$ & $1.27(1.02-1.57)$ \\
\hline$\geq 28.0$ & $213(7.34)$ & $64(30.05)$ & $1.68(1.20-2.35)$ \\
\hline \multicolumn{4}{|l|}{ Marital status } \\
\hline Married & $2249(77.42)$ & $491(21.83)$ & 1.00 \\
\hline Unmarried & $545(18.76)$ & $117(21.47)$ & $0.82(0.58-1.15)$ \\
\hline Divorced/separated/widowed & $111(3.82)$ & $36(32.43)$ & $1.48(0.94-2.33)$ \\
\hline
\end{tabular}


Table 1. Continued

\begin{tabular}{|c|c|c|c|}
\hline \multirow{3}{*}{ Characteristics } & \multirow{3}{*}{ Subjects (n [\%]) } & \multicolumn{2}{|c|}{ Subjects with EDS (ESS > 10) } \\
\hline & & \multirow{2}{*}{$\mathrm{n}(\%)$} & \multirow{2}{*}{$\begin{array}{c}\text { Multivariate analysis } \\
\text { OR }(95 \% \mathrm{CI})\end{array}$} \\
\hline & & & \\
\hline \multicolumn{4}{|l|}{ Education } \\
\hline Primary school or lower & $424(14.59)$ & $124(29.25)$ & $1.68(1.27-2.24)$ \\
\hline Secondary/high school & $1841(63.35)$ & $348(18.90)$ & 1.00 \\
\hline University or higher & $641(22.06)$ & $172(26.83)$ & $1.68(1.29-2.17)$ \\
\hline \multicolumn{4}{|l|}{ Occupation } \\
\hline Office worker & $754(26.01)$ & $169(22.41)$ & 1.00 \\
\hline Manual worker & $1576(54.36)$ & $349(22.14)$ & $1.04(0.82-1.34)$ \\
\hline Student & $131(4.52)$ & $28(21.37)$ & $1.01(0.60-1.70)$ \\
\hline Other & $438(15.11)$ & $95(21.69)$ & $1.04(0.76-1.42)$ \\
\hline \multicolumn{4}{|l|}{ Family income (Yuan/mo) } \\
\hline$<2000$ & $1415(48.86)$ & $327(23.11)$ & $1.18(0.96-1.45)$ \\
\hline $2000-5000$ & $1219(42.09)$ & $251(20.59)$ & 1.00 \\
\hline$\geq 5000$ & $262(9.05)$ & $63(24.05)$ & $1.04(0.75-1.46)$ \\
\hline \multicolumn{4}{|l|}{ Smoker status } \\
\hline Non-smoker & $2024(69.65)$ & $443(21.89)$ & 1.00 \\
\hline Current smoker & $792(27.25)$ & $178(22.47)$ & $1.14(0.87-1.50)$ \\
\hline Ex-smoker & $90(3.10)$ & $23(25.56)$ & $1.12(0.65-1.93)$ \\
\hline \multicolumn{4}{|l|}{ Alcohol consumption } \\
\hline Never & $2264(77.93)$ & $491(21.69)$ & 1.00 \\
\hline Yes & $641(22.07)$ & $153(23.87)$ & $1.11(0.86-1.43)$ \\
\hline \multicolumn{4}{|l|}{ Exercise Status } \\
\hline Never & $468(16.15)$ & $107(22.86)$ & $0.91(0.66-1.25)$ \\
\hline Less than 4 times/mo & $290(10.01)$ & $76(26.21)$ & $1.03(0.73-1.46)$ \\
\hline At least once/wk & $452(15.60)$ & $111(24.56)$ & 1.00 \\
\hline At least once/day & $1687(58.23)$ & $348(20.63)$ & $0.82(0.63-1.06)$ \\
\hline \multicolumn{4}{|c|}{ Family history of gastrointestinal tumors or diseases } \\
\hline None & $2603(89.60)$ & $566(21.74)$ & 1.00 \\
\hline Yes & $302(10.40)$ & $78(25.83)$ & $1.26(0.95-1.68)$ \\
\hline \multicolumn{4}{|l|}{ Chronic diseases } \\
\hline Liver disease & $141(4.85)$ & $40(28.37)$ & $1.23(0.82-1.83)$ \\
\hline Hypertension & $342(11.77)$ & $89(26.02)$ & $1.01(0.74-1.38)$ \\
\hline Myocardial infarction & $11(0.38)$ & $4(36.36)$ & $1.37(0.37-5.06)$ \\
\hline Angina pectoris & $43(1.48)$ & $13(30.23)$ & $1.17(0.57-2.42)$ \\
\hline Cerebrovascular disease & $71(2.44)$ & $21(29.58)$ & $1.28(0.72-2.28)$ \\
\hline Chronic bronchitis & $117(4.03)$ & $38(32.48)$ & $1.37(0.89-2.11)$ \\
\hline Asthma & $31(1.07)$ & $16(51.61)$ & $3.03(1.42-6.44)$ \\
\hline Renal disease & $77(2.65)$ & $22(28.57)$ & $1.16(0.67-2.00)$ \\
\hline Osteoarthritis & $90(3.10)$ & $29(32.22)$ & $1.58(0.97-2.55)$ \\
\hline Rheumarthritis & $128(4.40)$ & $40(31.25)$ & $1.30(0.86-1.96)$ \\
\hline Diabetes & $95(3.27)$ & $24(25.26)$ & $1.06(0.64-1.75)$ \\
\hline Anxiety & $8(0.28)$ & $2(25.00)$ & $0.63(0.10-4.16)$ \\
\hline Depression & $12(0.41)$ & $5(41.67)$ & $2.65(0.74-9.47)$ \\
\hline
\end{tabular}

${ }^{a}$ Chinese body mass index (BMI) category was applied (18.5, 24.0, 28.0).

EDS, excessive daytime sleepiness; ESS, Epworth Sleepiness Scale. 
marital status, occupation, family income, smoke status, alcohol consumption, and exercise status. Though OR values of family history of gastrointestinal tumors or diseases and all chronic diseases were above 1.00 , most of them were not significantly associated with EDS, except for asthma (OR, 3.03; 95\% CI, 1.42-6.44).

\section{Features of Excessive Daytime Sleepiness in Different Subtypes of Functional Gastrointestinal Disorders}

Figure shows the median (Q1, Q3) ESS score in different types of FGIDs. Median ESS score of gallbladder dysfunction

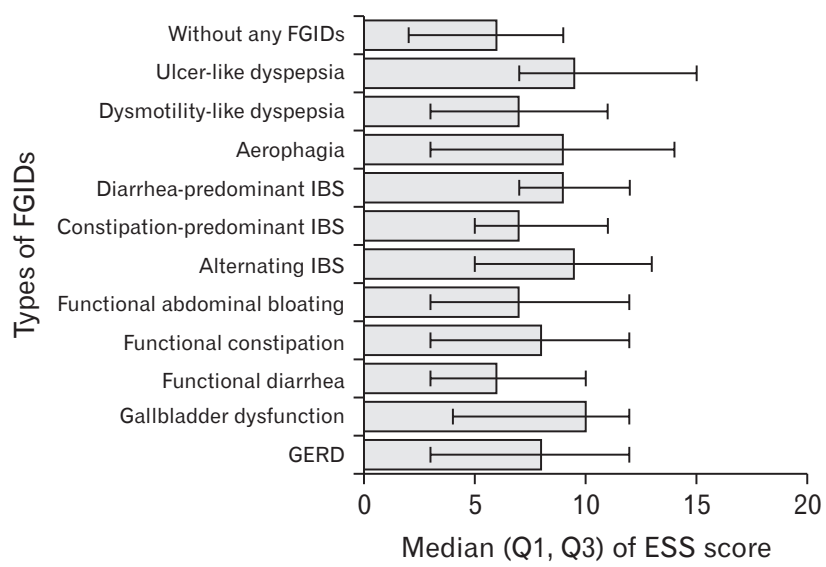

Figure. Median (Q1, Q3) of Epworth Sleepiness Scale (ESS) score in different types of functional gastrointestinal disorders (FGIDs). IBS, irritable bowel syndrome; GERD, gastroesophageal reflux disease. was the highest among FGIDs, while functional diarrhea was the lowest. In the 2 types of dyspepsia defined by the Rome II criteria, EDS was more severe in ulcer-like dyspepsia than in dysmotilitylike dyspepsia. Among the 3 types of IBS, EDS became more moderate in the decreasing order of alternating IBS, diarrheapredominant IBS, and constipation-predominant IBS.

The association between FGIDs and EDS is shown in Table 2. We selected meaningful demographic characteristics from all the demographic characteristics listed in Table 1 with stepwise method using multiple logistic regression. After selection, reserved demographic characteristics were BMI level, education, asthma, osteoarthritis, family history of gastrointestinal tumors or diseases, and chronic bronchitis. After adjustment of these reserved demographic characteristics and anxiety/depression, significant independent predictors of EDS were ulcer-like dyspepsia (OR, 2.50; 95\% CI, 1.08-5.79), diarrhea-predominant IBS (OR, 2.00; 95\% CI, 1.093.66), alternating IBS (OR, 2.32; 95\% CI, 1.30-4.13), functional constipation (OR, 1.68; 95\% CI, 1.20-2.35), and GERD (OR, $1.72 ; 95 \% \mathrm{CI}, 1.08-2.72)$. We did not observe significant association between EDS and dysmotility-like dyspepsia, aerophagia, constipation-predominant IBS, functional abdominal bloating, functional diarrhea, and gallbladder dysfunction.

\section{Impacts of the Number of Functional Gastrointestinal Disorders on Excessive Daytime Sleepiness}

The association between the number of FGIDs and EDS is

Table 2. Association Between Functional Gastrointestinal Disorders and Excessive Daytime Sleepiness

\begin{tabular}{|c|c|c|c|c|}
\hline \multirow{3}{*}{ Functional gastrointestinal disorders } & \multirow{3}{*}{ Subjects (n [\%]) } & \multicolumn{3}{|c|}{ Subjects with EDS (ESS > 10) (n [\%]) } \\
\hline & & \multirow{2}{*}{ (n [\%]) } & \multicolumn{2}{|c|}{ Multivariate analysis } \\
\hline & & & $\mathrm{OR}^{\mathrm{a}}(95 \% \mathrm{CI})$ & $P$-value \\
\hline Ulcer-like dyspepsia & $24(0.83)$ & $11(1.71)$ & $2.50(1.08,5.79)$ & 0.032 \\
\hline Dysmotility-like dyspepsia & $47(1.62)$ & $13(2.02)$ & $1.40(0.72,2.72)$ & 0.319 \\
\hline Aerophagia & $51(1.75)$ & $22(3.42)$ & $1.69(0.93,3.24)$ & 0.080 \\
\hline Diarrhea-predominant IBS & $53(1.82)$ & $20(3.11)$ & $2.00(1.09,3.66)$ & 0.024 \\
\hline Constipation-predominant IBS & $43(1.48)$ & $11(1.71)$ & $1.08(0.52,2.26)$ & 0.828 \\
\hline Alternating IBS & $54(1.86)$ & $22(3.42)$ & $2.32(1.30,4.13)$ & 0.004 \\
\hline Functional abdominal bloating & $157(5.40)$ & $48(7.45)$ & $1.34(0.92,1.97)$ & 0.131 \\
\hline Functional constipation & $191(6.57)$ & $64(9.94)$ & $1.68(1.20,2.35)$ & 0.003 \\
\hline Functional diarrhea & $45(1.55)$ & $9(1.40)$ & $0.79(0.37,1.67)$ & 0.534 \\
\hline Gallbladder dysfunction & $15(0.52)$ & $7(1.09)$ & $2.31(0.76,7.02)$ & 0.139 \\
\hline GERD & $94(3.23)$ & $37(5.75)$ & $1.72(1.08,2.72)$ & 0.022 \\
\hline
\end{tabular}

${ }^{a}$ ORs were adjusted by the following demographic characteristics: body mass index level, education, asthma, osteoarthritis, family history of gastrointestinal tumors or diseases, and chronic bronchitis, which were selected by stepwise method using multiple logistic regression. Though not been selected, anxiety, and depression were still adjusted due to they might act as potential confounders.

EDS, excessive daytime sleepiness; ESS, Epworth Sleepiness Scale; IBS, irritable bowel syndrome; GERD, gastroesophageal reflux disease.

$P$-value $<0.05$ is significant. 
Table 3. Association Between the Number of Functional Gastrointestinal Disorders and Excessive Daytime Sleepiness

\begin{tabular}{|c|c|c|c|c|}
\hline \multirow{3}{*}{ Number of FGIDs } & \multirow{3}{*}{ Subjects (n [\%]) } & \multicolumn{3}{|c|}{ Subjects with EDS (ESS > 10) (n [\%]) } \\
\hline & & \multirow{2}{*}{$(\mathrm{n}[\%])$} & \multicolumn{2}{|c|}{ Multivariate analysis } \\
\hline & & & $\mathrm{OR}^{\mathrm{a}}(95 \% \mathrm{CI})$ & $P$-value \\
\hline 0 & $2296(79.01)$ & $447(69.41)$ & 1.00 & - \\
\hline 1 & $471(16.21)$ & $143(22.20)$ & $1.72(1.37-2.15)$ & $<0.001$ \\
\hline 2 & $117(4.03)$ & $43(6.68)$ & $2.43(1.63-3.62)$ & $<0.001$ \\
\hline$\geq 3$ & $22(0.76)$ & $11(1.71)$ & $3.26(1.37-7.78)$ & 0.008 \\
\hline
\end{tabular}

${ }^{a}$ ORs were adjusted by the following demographic characteristics: body mass index level, education, asthma, osteoarthritis, family history of gastrointestinal tumors or diseases, and chronic bronchitis, which were selected by stepwise method using multiple logistic regression. Though not been selected, anxiety and depression were still adjusted due to they might act as potential confounders.

EDS, excessive daytime sleepiness; ESS, Epworth Sleepiness Scale; FGIDs, functional gastrointestinal disorders.

$P$-value $<0.05$ is significant.

presented in Table 3. The proportion of those with only one kind of FGIDs in the subjects with EDS (22.20\%) was significantly higher than in those without EDS (14.50\%) (OR, 1.72; 95\% CI, 1.37-2.15). Besides, OR rose along with the increasing number of FGIDs: with 2 FGIDs (OR, 2.43; 95\% CI, 1.63-3.62); with 3 FGIDs or more (OR, 3.26; 95\% CI, 1.37-7.78).

\section{Discussion}

EDS is common in the general Chinese population and is associated with the impairment of HRQOL. ${ }^{6}$ The reason why the prevalence of EDS is so high in China has been discussed previously, which might be a result of increase in obesity and overweight in Mainland China, extensive use of electronic products, and a cultural peculiarity of taking afternoon naps of the Chinese. ${ }^{6}$ Relationship between GERD and EDS has been reported before, poor sleep quality and sleep disruption were found to be associated with IBS and FD according to previous studies, which indicated that sleep status that was closely connected to gastrointestinal functions and impaired sleep may contribute to FGIDs..$^{10-12,16,26-28}$ However, studies systematically evaluating relationships between FGIDs and EDS are still insufficient. In our study, ulcer-like dyspepsia, diarrhea-predominant IBS, alternating IBS, functional constipation, and GERD were found to be significantly associated with EDS, which was in consistency with a similar previous study. ${ }^{17}$

Furthermore, we applied the Rome II criteria and RDQ to diagnose FGIDs so that we were able to divide FD and IBS into more specific subtypes in order to avoid confusion of similar diseases, such as diarrhea-predominant IBS and functional diarrhea, constipation-predominant IBS, and functional constipation. ${ }^{29,30}$

Overweight and obesity were significantly associated with EDS. Obesity has been proved to be a major risk factor for EDS, while weight loss is associated with its remission. ${ }^{31}$ A major cause of EDS is poor sleep quality, which is tightly associated with obstructive sleep apnea syndrome (OSAS), and OSAS is supposed to result from and contribute to obesity. ${ }^{4,32}$ On the other hand, severe obesity was also considered to be directly associated with EDS and sleep disorders in the absence of OSAS. ${ }^{33}$ Many studies have demonstrated that EDS correlateds with asthma in both children and adults, which is in consistence with our research. Asthma and OSAS are detrimental to each other through various mechanisms, and asthma may contribute to EDS through OSAS and other potential mechanisms. ${ }^{34}$ The prevalence of anxiety/depression was too low, it might be related to retrospective evaluation of these symptoms or not-validated tool for detection of these symptoms. However, anxiety and depression were still adjusted in Table 2 and Table 3 since they might act as potential confounders.

Dysregulation of the brain-gut axis has been suggested as an important factor in the pathogenesis of FGIDs. ${ }^{35}$ The autonomic nervous system and endocrine factors may mediate abnormal braingut interactions in IBS, while autonomic dysregulation also has been discussed as a cause of daytime sleepiness, which may help to explain the high prevalence of EDS in IBS patients. ${ }^{36,37}$ Specific IBS subgroups had different patterns of autonomic and endocrine functioning. The constipation-predominant subgroup is associated with a cholinergic abnormality and the diarrhea-predominant subgroup with an adrenergic abnormality. ${ }^{38}$ Diarrhea-predominant subgroup also showed a significant postprandial increase in cortisol, which was not evident in constipation-predominant subgroups. ${ }^{39}$ The differences between the 2 subgroups of IBS above may provide an explanation for our result that diarrhea-predominant IBS was significantly associated with EDS while the constipation-predominant IBS was not. However, more detailed cause-effect relationship between the different subtypes of IBS and EDS deserves investiga- 
tion.

The Rome II criteria classifies FD into ulcer-like dyspepsia and dysmotility-like dyspepsia, while it classified FD into the epigastric pain syndrome (EPS) and postprandial distress syndrome (PDS). ${ }^{40}$ It was indicated that PDS other than EPS was associated with sleep disturbance according to a Chinese research. ${ }^{41}$ In our study, ulcerlike dyspepsia other than dysmotility-like dyspepsia was still significantly associated with EDS after adjustment for anxiety, depression, and other confounders. However, since anxiety and depression were all self-reported in our study, the association between FD (defined as Rome III criteria) and EDS needs further studies, in which possible confounders like psychological distress and obesity should be better controlled.

Functional constipation was found to be associated with EDS. Interestingly, EDS and constipation are both non-motor symptoms perceived more than 10 years before motor symptoms in Parkinson's disease, latent links may exist in the coexistence of functional constipation and sleepiness. ${ }^{42}$ Although aerophagia did not enter the final multiple regression (OR, 1.69; 95\% CI, 0.93-3.24), its lower boundary of the confidence interval of OR was close to 1 . Further studies about whether aerophagia was associated with EDS need to be addressed. Prevalence of functional abdominal bloating in individuals with EDS was $7.45 \%$, but it did not enter the final multiple regression, which might be explained by the fact that it is common commodity of other gastrointestinal disorders such as IBS and constipation. ${ }^{43}$ Gallbladder dysfunction proved not to be a risk factor of EDS. Gallbladder dysfunction was common in IBS patients, which might be an explanation. ${ }^{44}$ We observed that OR of EDS increased directly with the increasing numbers of digestive system diseases, indicating that the risk of EDS was related to the numbers of FGIDs. The relationships between FGIDs and sleep problems are complex, and coexistence of FGIDs may be associated with more severe sleep problems. Putative brain gut interaction may be involved in sleep problems, GERD and FGIDs. ${ }^{45}$ Physicians should pay attention to the sleep status of patients who are troubled with more than one kind of FGIDs.

The strength of this study was that the sample covered 5 main regions and may represent a general Chinese population, and also, the survey achieved a high response rate that minimized potential bias for responder bias. These advantages provided presentative, generalizable population-based, and high-quality data which was able to present the real association between EDS and FGIDs. Furthermore, global consensus-based definitions of FGIDs and GERD were used, and reliability and validation of Chinese version of ESS were confirmed. ${ }^{6,22,46}$ However, this study was a cross-sectional study and these types of studies are insufficient in establishing cause-effect relationships. Also, the prevalence of EDS found in this study might be overestimated because the ESS is a subjective measuring method. ${ }^{6}$

Financial support: The study was supported by the National Nature Science Foundation of China (Grant No. 81502895, 81373105, and 81502880) and the Fourth Round of Three-year Action Plan on Public Health Discipline and Talent Program: Evidence-based Public Health and Health Economics (Grant No. 15GWZK0901).

\section{Conflicts of interest: None.}

Author contributions: Jia $\mathrm{He}$ is the guarantor of this article and contributed to the critical revision of manuscript; Sicheng $\mathrm{Wu}$, Shuqi Chen, and Yangfang Zhao contributed equally to data analysis, drafting of manuscript, and critical revision of manuscript; and Xiuqiang Ma and Rui Wang contributed to study design, field work, quality control, and data collection.

\section{References}

1. Hublin C, Kaprio J, Partinen M, Heikkila K, Koskenvuo M. Daytime sleepiness in an adult, Finnish population. J Intern Med 1996;239:417423.

2. Ohayon MM, Caulet M, Philip P, Guilleminault C, Priest RG. How sleep and mental disorders are related to complaints of daytime sleepiness. Arch Intern Med 1997;157:2645-2652.

3. Hara C, Lopes Rocha F, Lima-Costa MF. Prevalence of excessive daytime sleepiness and associated factors in a Brazilian community: the Bambui study. Sleep Med 2004;5:31-36.

4. Bixler EO, Vgontzas AN, Lin HM, Calhoun SL, Vela-Bueno A, Kales A. Excessive daytime sleepiness in a general population sample: the role of sleep apnea, age, obesity, diabetes, and depression. J Clin Endocrinol Metab 2005;90:4510-4515.

5. Kaneita Y, Ohida T, Uchiyama M, et al. Excessive daytime sleepiness among the Japanese general population. J Epidemiol 2005;15:1-8.

6. Wu S, Wang R, Ma X, Zhao Y, Yan X, He J. Excessive daytime sleepiness assessed by the Epworth Sleepiness Scale and its association with health related quality of life: a population-based study in China. BMC Public Health 2012;12:849.

7. Melamed S, Oksenberg A. Excessive daytime sleepiness and risk of occupational injuries in non-shift daytime workers. Sleep 2002;25:315-322.

8. Roth T, Roehrs TA. Etiologies and sequelae of excessive daytime sleepiness. Clin Ther 1996;18:562-576; discussion 561.

9. Stroe AF, Roth T, Jefferson C, et al. Comparative levels of excessive daytime sleepiness in common medical disorders. Sleep Med 2010;11:890-896.

10. Wells MM, Roth L, Chande N. Sleep disruption secondary to overnight call shifts is associated with irritable bowel syndrome in residents: a crosssectional study. Am J Gastroenterol 2012;107:1151-1156. 
11. Miwa H. Life style in persons with functional gastrointestinal disorders-large-scale internet survey of lifestyle in Japan. Neurogastroenterol Motil 2012;24:464-471, e217.

12. Nicholl BI, Halder SL, Macfarlane GJ, et al. Psychosocial risk markers for new onset irritable bowel syndrome--results of a large prospective population-based study. Pain 2008;137:147-155.

13. Kumar M, Gyawali CP, Cassell B, Nix BD, Sayuk GS. Disturbed sleep predicts higher GI and somatic symptom burdens in irritable bowel syndrome (IBS). Gastroenterology 2011;140:S-464.

14. Lacy BE, Everhart K, Crowell MD. Functional dyspepsia is associated with sleep disorders. Clin Gastroenterol Hepatol 2011;9:410-414.

15. Yamawaki H, Futagami S, Shimpuku M, et al. Impact of sleep disorders, quality of life and gastric emptying in distinct subtypes of functional dyspepsia in Japan. J Neurogastroenterol Motil 2014;20:104-112.

16. Wang R, Zou D, Ma X, et al. Impact of gastroesophageal reflux disease on daily life: the Systematic Investigation of Gastrointestinal Diseases in China (SILC) epidemiological study. Health Qual Life Outcomes 2010;8:128.

17. Morito Y, Aimi M, Ishimura N, et al. Association between sleep disturbances and abdominal symptoms. Intern Med 2014;53:2179-2183.

18. Rotem AY, Sperber AD, Krugliak P, Freidman B, Tal A, Tarasiuk A. Polysomnographic and actigraphic evidence of sleep fragmentation in patients with irritable bowel syndrome. Sleep 2003;26:747-752.

19. Yan X, Wang R, Zhao Y, et al. Systematic investigation of gastrointestinal diseases in China (SILC): validation of survey methodology. BMC Gastroenterol 2009;9:86.

20. Zhou BF; Cooperative Meta-Analysis Group of the Working Group on Obesity in China. Predictive values of body mass index and waist circumference for risk factors of certain related diseases in Chinese adults--study on optimal cut-off points of body mass index and waist circumference in Chinese adults. Biomed Environ Sci 2002;15:83-96.

21. Johns MW. A new method for measuring daytime sleepiness: the Epworth sleepiness scale. Sleep 1991;14:540-545.

22. Kwan AC, Bao T, Chakkaphak S, et al. Validation of Rome II criteria for functional gastrointestinal disorders by factor analysis of symptoms in Asian patient sample. J Gastroenterol Hepatol 2003;18:796-802.

23. Talley NJ, Stanghellini V, Heading RC, Koch KL, Malagelada JR, Tytgat GN. Functional gastroduodenal disorders. Gut 1999;45(suppl 2):II37-II42.

24. Kim JY, Kim N, Seo PJ, et al. Association of sleep dysfunction and emotional status with gastroesophageal reflux disease in Korea. J Neurogastroenterol Motil 2013;19:344-354.

25. Zhao Y, Zou D, Wang R, et al. Dyspepsia and irritable bowel syndrome in China: a population-based endoscopy study of prevalence and impact. Aliment Parmacol Ther 2010;32:562-572.

26. Futagami S, Shimpuku M, Yamawaki H, et al. Sleep Disorders in Functional Dyspepsia and Future Therapy. J Nippon Med Sch 2013;80:104109.

27. Bellini M, Gemignani A, Gambaccini D, et al. Evaluation of latent links between irritable bowel syndrome and sleep quality. World J Gastroenterol 2011;17:5089-5096.

28. Sisson G, Junghans C, Bjarnason I. Poor sleep quality in irritable bowel syndrome: a comparison with healthy adult controls. Gut 2010;59(suppl
1):A140.

29. Zhao YF, Guo XJ, Zhang ZS, et al. Epidemiology of functional diarrhea and comparison with diarrhea-predominant irritable bowel syndrome: a population-based survey in China. PloS One 2012;7:e43749.

30. Zhao YF, Ma XQ, Wang R, et al. Epidemiology of functional constipation and comparison with constipation-predominant irritable bowel syndrome: the Systematic Investigation of Gastrointestinal Diseases in China (SILC). Aliment Pharmacol Ther 2011;34:1020-1029.

31. Fernandez-Mendoza J, Vgontzas AN, Kritikou I, Calhoun SL, Liao D, Bixler EO. Natural history of excessive daytime sleepiness: role of obesity, weight loss, depression, and sleep propensity. Sleep 2015;38:351-360.

32. Wosu AC, Velez JC, Barbosa C, et al. The relationship between high risk for obstructive sleep apnea and general and central obesity: findings from a sample of Chilean college students. ISRN Obes 2014;2014:871681.

33. Resta O, Foschino Barbaro MP, Bonfitto P, et al. Low sleep quality and daytime sleepiness in obese patients without obstructive sleep apnoea syndrome. J Intern Med 2003;253:536-543.

34. Alkhalil M, Schulman E, Getsy J. Obstructive sleep apnea syndrome and asthma: what are the links? J Clin Sleep Med 2009;5:71-78.

35. American Gastroenterological Association. American Gastroenterological Association medical position statement: irritable bowel syndrome. Gastroenterology 2002;123:2105-2107.

36. Wood JD, Alpers DH, Andrews PL. Fundamentals of neurogastroenterology. Gut 1999;45(suppl 2):II6-II16.

37. Slater G, Steier J. Excessive daytime sleepiness in sleep disorders. J Thorac Dis 2012;4:608-616.

38. Aggarwal A, Cutts TF, Abell TL, et al. Predominant symptoms in irritable bowel syndrome correlate with specific autonomic nervous system abnormalities. Gastroenterology 1994;106:945-950.

39. Elsenbruch S, Orr WC. Diarrhea- and constipation-predominant IBS patients differ in postprandial autonomic and cortisol responses. Am J Gastroenterol 2001;96:460-466.

40. Tack J, Talley NJ. Functional dyspepsia--symptoms, definitions and validity of the Rome III criteria. Nat Rev Gastroenterol Hepatol 2013;10:134141.

41. Fang YJ, Liou JM, Chen CC, et al. Distinct aetiopathogenesis in subgroups of functional dyspepsia according to the Rome III criteria. Gut 2015;64:1517-1528.

42. Pont-Sunyer C, Hotter A, Gaig C, et al. The onset of nonmotor symptoms in Parkinson's disease (the ONSET PD study). Mov Disord 2015;30:229-237.

43. Sullivan SN. Functional abdominal bloating. J Clin Gastroenterol 1994;19:23-27.

44. Reddy I, Shankar A, Selby L, Dignan M, Shankar U. Association of gallbladder dysfunction and irritable bowel syndrome in an Appalachian population. Am J Gastroenterol 2013;108:S565.

45. Jung HK, Choung RS, Talley NJ. Gastroesophageal reflux disease and sleep disorders: evidence for a causal link and therapeutic implications. J Neurogastroenterol Motil 2010;16:22-29.

46. Ho KY, Gwee KA, Khor JL, Selamat DS, Yeoh KG. Validation of a graded response questionnaire for the diagnosis of gastroesophageal reflux disease in an Asian primary care population. J Clin Gastroenterol $2008 ; 42: 680-686$. 